\title{
THE ICRC's SHORT-WAVE PROGRAMMES
}

The ICRC's first short-wave radio programmes went on the air in 1945. The original idea behind the scheme was to help thousands of families who had been split up during the Second World War to come together again. The radio broadcast the names of those reported missing, prisoners of war and civilian prisoners, who were listed on the files of the ICRC's Central Tracing Agency. The Swiss broadcasting authorities then granted the ICRC special short-wave frequencies which were used periodically until 1965 .

This was the year that marked the start of regular collaboration between the ICRC and the Swiss short-wave service, in the form of two-monthly "experimental programmes of the Red Cross". These radio programmes were always referred to by their English name, the "Red Cross Broadcasting Service" (RCBS); each lasted for fifty-five minutes, and gave news on the Red Cross in five languages (French, English, Spanish, German and Arabic). They could be picked up in Europe, North America and the Near East.

One of the reasons for launching these experimental programmes of the Red Cross lay in the fact that the ICRC needed to be certain of its constant ability to reach the farthest-flung parts of the world by radio, if the situation so required. In this way, it could make sure that any urgent humanitarian messages had been received as intended, under acceptable technical conditions.

But the ICRC capitalized on the powerful, rapid and far-reaching nature of the broadcasting medium by informing a wide audience about the Red Cross, its organization and activities, the ICRC, humanitarian law, and how listeners could help.

Thus, since 1965, the ICRC's short-wave programmes have not been devoted solely to news, but have also become a means of informing the general public about the Red Cross and its point of view.

In 1971, the ICRC requested the help of the Swiss broadcasting authorities in reaching an even wider audience, by beaming its pro- 
grammes to the Middle East, Africa and the Far East. The first programme on these wavelengths went out in November of the same year. As a result, the number and variety of potential listeners became so great that it was difficult to cater properly for all. Nonetheless, this did not constitute a major problem for the Red Cross, which aims at being -and is in fact-universal. The subject-matter of the radio programmes is universal: only the language changes. Audience survey reports from all over the world were quick to demonstrate the interest expressed by listeners, and this encouraged the ICRC to continue these information programmes, and to make continual efforts to improve them.

Over the past few years, the ICRC Broadcasting Service has concentrated on describing the institution's worldwide activities, no longer simply by reading out news bulletins, but by means of first-hand accounts from delegates, reports from areas where there was fighting, and analyses of the situation by specialists at headquarters in Geneva. It has made special efforts to obtain the widest possible variety of impressions and eye-witness reports from people of different nationalities or who played very different roles in a given humanitarian relief operation. From a more educational angle, subjects dealt with have included humanitarian law, political prisoners, and torture.

More recently, the scope of the programmes has been widened to include the Red Cross movement as a whole: the two-monthly broadcasts on Swiss short-wave now include a regular feature on the activities of the League of Red Cross Societies and those of the National Red Cross, Red Crescent and Red Lion and Sun Societies.

One of the main concerns of the Broadcasting Service is to establish a more meaningful two-way exchange of information and ideas with its listeners. Without this kind of communication, progress is impossible. Every programme therefore contains a request that listeners send in their criticisms and comments.

In 1977, as a direct result of suggestions received from many listeners, a number of changes were introduced in the structure of RCBS programmes for Europe, North America and the Middle East. Instead of broadcasting the same programme on the three days allocated to the RCBS every two months - the last Monday, Wednesday and Friday of the month-it has now become possible to change the programme content on each of these three days. On Mondays, the language used is English, on Wednesdays French and German, and on Fridays Spanish and Arabic. Each fifty-five minute programme is transmitted on the $7210 \mathrm{KHz}$ frequency / 41.60 metre waveband, at four different moments in the day: 6 a.m., 11.30 a.m., 5 p.m. and 10 p.m. GMT. 
The ICRC Broadcasting Service receives a constant flow of letters from listeners in all parts of the globe, in five different languages. Each should be answered individually, and no question should be ignored. Since the staff of the ICRC radio team numbers only two, a considerable number of replies are given directly over the air. There is a two-fold advantage in this: it simplifies correspondence and, at the same time, provides meaningful material for programmes on subjects requested by the listeners themselves.

Even on a limited scale, this kind of dialogue with men and women so far away, so different from each other and yet so alike, is fundamental to making known the humanitarian ideals of the Red Cross. There can be no doubt that the simple, direct and effective medium of radio broadcasting is ideal for reaching out to people, bringing them together and encouraging them to go further.

M. $M$. 